\title{
ESTIMATIVA DOS COEFICIENTES ESPECIFICOS DE MORTALIDADE INFANTIL SEGUNDO PESO AO NASCER NO MUNICfPIO DE SÃO PAULO (BRASIL) *
}

Carlos Augusto Monteiro**

MONTEIRO, C. A. Estimativa dos coeficientes especificos de mortalidade infantil segundo peso ao nascer no municipio de são Paulo (Brasil). Rev. Saúde públ., S. Paulo, $1.5: 603-10,1981$.

RESUMO: Foi estimada a distribuição do peso ao nascer da coorte dos recém-nascidos que deu origem aos obitos infantis estudados pela Investigação Interamericana de Mortalidade na Infância no projeto de São Paulo (1968-70) e determinados os riscos de óbito associados a cada intervalo de peso de nascimento. Assim, foram apurados coeficientes de mortalidade infantil de $305,5,50,2$ e 34,4 para recém-nascidos de baixo peso, peso deficiente e peso superior a $3.000 \mathrm{~g}$. A comparação destes coeficientes com os registrados na área americana incluída na investigação citada (Califómia), revelou excesso de mortalidade, particularmente notável não para os recém-nascidos de baixo peso, mas para os recém-nascidos de peso superior a 3.000 g. O ajuste da mortalidade de São Paulo (Brasil) à distribuição do peso ao nascer observada na Califórnia foi capaz de explicar $15 \%$ do excesso da mortalidade infantil e $21 \%$ do excesso da mortalidade neonatal de São Paulo.

UNITERMOS: Mortalidade infantil. Peso ao nascer. São Paulo, SP, Brasil.

\section{INTRODUÇÃO}

Entre junho de 1968 e maio de 1970, a Investigação Interamericana de Mortalidade na Infância ${ }^{15}$ estudou, de forma exaustiva, as causas dos óbitos de menores de 5 anos na área do municipio de São Paulo. O estudo foi baseado em uma amostra dos óbitos de residentes no distrito de São Paulo, cuja população compreendia aproximadamente $90 \%$ da população do município. Uma das causas então apontadas como das mais importantes em determinar de forma direta ou indireta o óbito infantil foi o baixo peso ao nascer. Naquela ocasião estimou-se que $60,9 \%$ das crianças de São Paulo que morreram antes de completar 28 dias de idade haviam nascido com peso igual ou inferior a $2.500 \mathrm{~g}$. Este achado, associado à particularmente elevada mortalidade neonatal encontrada em São Paulo, uma das mais altas do continente, levou a que se atribuísse ao peso ao nascer e, consequientemente, à nutrição materna papel extremamente importante na determinação dos altos níveis de mortalidade infantil então registra-

* Financiado pela Fındação de Amparo à Pesquisa do Estado de São Paulo (FAPESP) - Processo $78 / 1109$.

* Do Departamento de Nutrição da Faculdade de Saúde Pública da USP - Av. Dr. Arnaldo, 715 - 01255 - São Paulo, SP - Brasil. 
MONTEIRO, C.A. Estimativa dos coeficientes específicos de mortalidade infantil segundo peso ao nascer no município de São Paulo (Brasil). Rev. Saúde públ., S. Paulo, 15:603-10, 1981.

dos no municipio 2. Neste contexto, e diante do aparente sucesso obtido por Lechtig e col. + em incrementar o peso ao nascer em aldeias da Guatemala por meio de suplementação calórica à gestante, a Secretaria de Estado da Saúde de São Paulo decide, a partir de 1977, alterar sua programação materno-infantil, introduzindo no pré-natal a suplementação alimentar ${ }^{3}$. Tal atitude foi seguida também pelas unidades sanitárias do município que, assim como as unidades do Estado, passaram a distribuir para as gestantes o suplemento calórico-protéico denominado "Gestal".

Deve-se ressaltar, entretanto, que a simples observação da incidência de recém-nascidos de baixo peso entre os óbitos infantis não permite aquilatar o impacto que aquela condição exerce sobre os niveis de mortalidade infantil. Para tanto é necessário que se conheça, concomitantemente, a distribuição do peso ao nascer do conjunto dos recém-nascidos vivos que originam os óbitos infantis. Infelizmente, no município de São Paulo, como na maioria das demais localidades incluídas na Investigação ${ }^{15}$, não se pôde conhecer, à época do estudo, a distribuição populacional dos pesos de nascimento.O objetivo deste trabalho será o de procurar estimar a distribuição do peso ao nascer da coorte dos recém-nascidos vivos do município de São Paulo que deu origem aos óbitos infantis estudados pela Investigação ${ }^{15} \mathrm{e}$, desta forma, poder determinar os riscos de óbito associados a cada intervalo de peso de nascimento. Conhecendo-se estes riscos e a distribuição da variável peso ao nascer, poder-se-á avaliar o quanto o desempenho desta variável é responsável pela excessiva mortalidade infantil observada no município de São Paulo.

\section{METODOLOGIA}

Para estimar a distribuição do peso ao nascer vigente no periodo 1960-70, utilizar-se-á um estudo apoiado en uma amostra probabilística dos partos hospitalares de nascidos-vivos ocorridos no municipio de
São Paulo, surgido tão somente em $1976^{11 .}$ É difícil averiguar a propriedade de a distribuição de 1976 traduzir a distribuição do período 1968-70. O acompanhamento dos valores reais do salário mínimo diante do custo de vida entre 1968 e 1976 mostra que - poder aquisitivo da população assalariadada se manteve estagnado entre os dois estudos 9 . Da mesma forma, o fato de o estudo de 1976 ter se baseado exclusivamente em nascimentos hospitalares năo deve prejudicar a projeção de seus resultados para o estudo de mortalidade de 1968-70, uma vez que no municipio de São Paulo e extremamente insignificante $o$ parto domiciliar ${ }^{16}$. A única sólida ressalva que parece existir para a transposição da distribuição de 1976 para o estudo de 1968-70 se deve a que este último estudo abrangeu apenas a área do distrito de São Paulo ( $90 \%$ da população do município) enquanto o estudo da distribuição do peso ao nascer de 1976 abrangeu também a população dos demais sete distritos que apresentam condiçōes de pobreza superiores às da média do município de São Paulo. Esta ressalva que opera, portanto, no sentido de superestimar a incidência de recém-nascidos de baixo peso na coorte 1968-70, deverá ser levada em conta na apreciação final dos resultados.

A justaposição da distribuição do peso ao nascer estimada para os nascidos vivos com a encontrada para os óbitos de menores de um ano permitirá que sejam calculados, para o período $1968-70$, os coeficientes de mortalidade infantil e neonatal para os recém-nascidos com $2.500 \mathrm{~g}$ ou menos (baixo peso ao nascer), com 2.501 a $3.000 \mathrm{~g}$ (peso deficiente) e com mais de $3.000 \mathrm{~g}$.

A avaliação do papel do peso ao nascer nos altos niveis de mortalidade infantil registrados no município de São Paulo, pela Investigação ${ }^{15}$, apoiat-se-á no ajuste direto 18 destes coeficientes a uma distribuição ideal de peso ao nascer. Esta será a distribuição observada no projeto da California nos EUA ${ }^{15}$, onde a Investigação registrou coeficientes de mortalidade infantil bastante 
MONTEIRO, C.A. Estimativa dos coeficientes específicos de mortalidade infantil segundo peso ao nascer no município de São Paulo (Brasil). Rev. Saúde públ., S. Paulo, 15:603-10, 1981.

baixos e que aqui serão tomados como padrão de referência para a mortalidade do município de São Paulo.

\section{RESULTADOS}

Estimativa dos coeficientes de mortalidade infantil no municipio de São Paulo segundo peso ao nascer

Segundo a Investigaçāo Interamericana de Mortalidade na Infância ${ }^{15}$, os 3.788 óbitos infantis por ela estudados corresponderam a 1.722 crianças que nasceram com baixo peso, 820 que nasceram com peso deficiente e 1.246 que nasceram com mais de $3.000 \mathrm{~g}$.

A coorte de nascimentos que deu origem a estes óbitos abrangeu, segundo a Investigação ${ }^{15}, 58.160$ nascidos vivos. A partir da projeção da distribuição do peso ao nascer observada em São Paulo em 1976 $\$, 69 \%$ de recém-nascidos de baixo peso, $28,07 \%$ de recém-nascidos de peso deficiente e $62,24 \%$ de recém-nascidos com mais de $3.000 \mathrm{~g}$ - pode-se estimar que esta coorte tenha correspondido a 5.636 recém-nascidos de baixo peso, 16.325 de peso deficiente $e$ 36.199 de peso superior a $3.000 \mathrm{~g}$.
A consideração das duas distribuições permite verificar que seriam de 305,5, 50,2 e 34,4, respectivamente, os coeficientes de mortalidade infantil para os recém-nascidos de baixo peso, de peso deficiente e de peso superior a $3.000 \mathrm{~g}$ (Tabela 1). Caracterizar-se-ia no municipio de São Paulo, portanto, un risco relativo de obito 8,8 vezes maior para os recém-nascidos de baixo peso com relação aos recém-nascidos com mais de $3.000 \mathrm{~g}$.

Do mesmo modo, a consideraçāo da distribuição do peso ao nascer entre os obitos neonatais mostra que seriam de $211,7,19,2$ e 12,5 , respectivamente, os coeficientes de mortalidade neonatal para os recém-nascidos de baixo peso, de peso deficiente e de peso superior a $3.000 \mathrm{~g}$ (Tabela 2).

Coeficientes de mortalidade infantil segundo peso ao nascer no municipio de Sáo Paulo e na Califórnia

A Investigação ${ }^{15}$ apurou para o municipic de São Paulo mortalidade infantil de 65,1 obitos para cada mil nascidos vivos. A mesma mortalidade apurada para a Califórnia foi de apenas 17,5 , revelando, por-

T A B E L A 1

Estimativa dos coeficientes de mortalidade infantil segundo o peso ao nascer na vigência da Investigação Interamericana de Mortalidade na Infância em São Paulo $(1968-70)$.

\begin{tabular}{cccc}
$\begin{array}{c}\text { Peso ao nascer } \\
\text { (gramas) }\end{array}$ & $\begin{array}{c}\text { Número de nascidos Número de óbitos } \\
\text { vivos (a) }\end{array}$ & $\begin{array}{c}\text { Coeficiente de } \\
\text { mortalidade } \\
\text { infantil (c) }\end{array}$ \\
\hline 2.500 & 5.636 & 1.722 & 305.5 \\
$2.501-3.000$ & 16.325 & 820 & 50.2 \\
$3.001 \mathrm{e}+$ & 36.199 & 1.246 & 34,4 \\
\hline Total & 58.160 & 3.788 & 65,1 \\
\hline
\end{tabular}

(a) Baseado na distribuição do peso ao nascer de 1976. Fonte: Monteiro e col.11 (1980).

(b) Estimados cle acordo com a distribuição do peso ao nascer de 2.742 óbitos. Fonte: Putfer e Serrano 1s (1973).

(c) óbitos de menores de 1 ano por mil nascidos viros. 
MONTEIRO, C.A. Estimativa dos coeficientes especificos de mortalidade infantil segundo peso ao nascer no município de São Paulo (Brasil), Rev. Saúde públ., S. Paulo, 15:603-10, 1981.

TA B E A 2

Estimativa dos coeficientes de mortalidade neonatal segundo o peso ao nascer na vigència da Investigação Interamericana de Mortalidade na Infância em São Paulo (1968-70).

\begin{tabular}{ccrc}
\hline $\begin{array}{c}\text { Peso ao nascer } \\
\text { (gramas) }\end{array}$ & $\begin{array}{c}\text { Número de nascidos Número de óbitos } \\
\text { vivos (a) }\end{array}$ & $\begin{array}{c}\text { Coeficiente de } \\
\text { mortalidade } \\
\text { neonatal (c) }\end{array}$ \\
\hline 2.500 & 5.636 & 1.193 & 211.7 \\
$2.501-3.000$ & 16.325 & 314 & 19,2 \\
$3.001 \mathrm{e}+$ & 36.199 & 451 & 12,5 \\
\hline Total & 58.160 & 1.958 & 33,7 \\
\hline
\end{tabular}

(a) Baseado na distribuição do peso ao nascer de 1976. Fonte: Monteiro e col.11 (1980).

(b) Estimados de acordo com a distribuição do peso ao nascer de 1.632 óbitos. Fonte: Puffer e Serrano 15 (1973).

(c) Ob:tos de menores de 28 dias por mil nascidos vivos.

tanto, para São Paulo um risco de óbito infantil 3,7 vezes maior.

A mortalidade infantil vista para cada intervalo de peso de nascimento mostra coeficientes sistematicamente mais altos para São Paulo (Tabela 3). Nota-se, entretanto, que enquanto a mortalidade dos recém-nascidos de baixo peso é 2,1 vezes mais alta em São Paulo do que na Califórnia, a mortalidade dos de peso deficiente é 4,2 vezes mais alta e a dos de peso superior a $3.000 \mathrm{~g}$ chega a ser 6,1 vezes mais alta. Caracteriza-se, portanto, um aumento do risco relativo de óbito infantil à medida que progride o peso de nascimento do recém-nascido de São Paulo.

Quanto aos coeficientes de mortalidade neonatal, a Investigação ${ }^{15}$ apurou 33,7 para o município de São Paulo e 12,7 para a Califórnia, revelando risco de óbito neonatal 2,6 vezes maior em São Paulo. A comparação destes coeficientes para cada intervalo de peso de nascimento mostra, também, coeficientes sistematicamente mais altos para o municipio de São Paulo e aponta, também, a existência de idêntico gradiente de risco relativo à medida que progride o peso ao nascer no município de São Paulo
(Tabela 3). Para os recém-nascidos de baixo peso, o risco de óbito neonatal é 1,6 vezes maior no município de São Paulo, para os de peso deficiente este risco é 3,6 vezes maior e para os nascidos com mais de $3.000 \mathrm{~g}$ o risco chega a ser 5,0 vezes maior.

Ajuste dos coeficientes de mortaludade infantil do municipio de São Paulo segundo a distribuição do peso ao nascer na Collifórnia

$\mathrm{Se}$ os coeficientes especificos de mortalidade infantil, vigentes no municipio de São Paulo, à época da Investigação ${ }^{15}$, incidissem sobre uma população de recém-nascidos com pesos de nascimento idênticos aos vigentes na Califórnia, o que ocorreria com a taxa de mortalidade infantil do município de São Paulo?

Uma mortalidade de 305,5 por mil incidiria sobre $7,6 \%$ dos recém-nascidos ao invés de incidir sobre 9,7\%. Uma mortalidade de 50,2 por mil incidiria sobre $191 \%$ dos recém-nascidos ao invés de incidir sobre $28,1 \%$. E, finalmente, por outro lado, uma mortalidade de 34,4 por mil incidiria sobre $73,3 \%$ dos recém-nascidos ao invés de incidir sobre $62,2 \%$. 
MONTEIRO, C.A. Estimativa dos coeficientes específicos de mortalidade infantil segundo peso ao nascer no municipio de São Paulo (Brasil). Rev. Saúde púbt., S. Paulo, 15:603-10, 1981.

T A B E L A 3

Coeficientes de mortalidade (a) segundo peso ao nascer no município de São Paulo e na Califórnia (b) (1968-70).

\begin{tabular}{|c|c|c|c|c|c|c|c|}
\hline \multirow{2}{*}{\multicolumn{2}{|c|}{ Peso ao nascer (gramas) }} & \multicolumn{3}{|c|}{ Mortalidade infantil } & \multicolumn{3}{|c|}{ Mortalidade neonatal } \\
\hline & & São Paulo & & lifórnia & São $\mathrm{Pa} \cdot \mathrm{lc}$ & & Califórnia \\
\hline & $\leqslant 2.500$ & 305,5 & - & 146,0 & 211,7 & - & 129,9 \\
\hline & $2.501-3.000$ & 50,2 & - & 11,8 & 19,2 & - & 5,4 \\
\hline & $3.001 \mathrm{e}+$ & 34.4 & 一 & 5,6 & 12,5 & - & 2,5 \\
\hline Total & & 65,1 & 一 & 17,3 & 33,7 & - & 12,7 \\
\hline
\end{tabular}

(a) obitos por mil nascidos riros.

(b) Fonte Puffer e Serrano 1.s (1973).

Desta forma, a mortalidade infantil do município de São Paulo cairia de 65,1 para 58,0 por mil nascidos vivos, se no município os recém-nascidos passassem a nascer com pesos idênticos aos observados na Califórnia. Neste caso haveria uma redução de $11 \%$ no coeficiente de mortalidade infantil do município de São Paulo, o que faria com que o diferencial de mortalidade São Paulo - Califórnia passasse de $65,1-17,3$ para 58,0 - 17,3. Ou seja, pode-se dizer que as diferenças existentes na composiçāo do peso ao nascer explicam $15 \%$ do excesso de mortalidade infantil do município de São Paulo frente à Califórnia.

A mesma suposição levaria a mortalidade neonatal do município de Sāo Paulo de 33,7 para 28,9 por mil nascidos vivos. A redução obtida seria de $7 \%$ e o diferencial de mortalidade São Paulo - Califórnia iria de 33,7 - 12,7 para 28,9 - 12,7, ou seja as diferenças existentes na composição do peso ao nascer explicariam $23 \%$ do excesso da mortalidade neonatal do município de São Paulo.

\section{COMENTARIOS}

Há duas questões que o presente estudo pretende responder:
1. Como, no município de São Paulo, relacionam-se peso ao nascer e probabilidade de óbito infantil?

2. Como, no municipio de São Paulo, o desempenho do peso ao nascer influi sobre a elevada mortalidade infantil?

Quanto à primeira questão, o estudo mostra inicialmente uma clara dependència entre probabilidade de óbito no primeiro ano de vida e peso ao nascer. Mesmo sem considerar outros fatores, percebe-se que, cado o elevadissimo risco de óbito que acompanha o recém-nascido de baixo peso, dificilmente outro fator isolado poderia influir de maneira mais decisiva sobre a sorte do recém-nascido no primeiro ano de vida. Neste particular, os resultados do municipio de São Paulo assemelham-se bastante a outros resultados observados tanto em localidades desenvolvidas $i .14$ como em localidades não desenvolvidas ${ }^{6,19}$.

Examinando, no entanto, mais detidamente os coeficientes de mortalidade segundo peso ao nascer no município de São Paulo, surgem alguns aspectos que parecen diferenciar a relação óbito infantil-peso ao nascer nesta localidade.

Inicialmente, pode-se notar que o risco relativo de óbito infantil que acompanha 
MONTEIRO, C.A. Estimativa dos coeficientes especificos de mortalidade infantil segundo peso ao nascer no municipio de São Paulo (Brasil). Rev. Saúde públ., S. Paulo, 15:603-10, 1981.

o recém-nascido de baixo peso no município de São Paulo, apesar de bastante importante, chegando a 8,8 em relação ao risco associado ao peso ao nascer superior a $3.000 \mathrm{~g}$, é bastante inferior ao mesmo risco observado na Califórnia que é de 26,1. Por outro lado, na comparação dos coeficientes especificos de mortalidade destas duas localidades, é exatamente o risco de óbito de quem nasce no municipio de São Paulo com mais de $3.000 \mathrm{~g}$ aquele que mais distancia São Paulo da Califórnia.

Com diferentes proporções, repetem-se as mesmas observações quando contrapõem-se os coeficientes específicos do município de São Paulo aos de uma localidade igualmente não desenvolvida como Ribeirão Preto, no Estado de São Paulo 19. O risco relativo de óbito infantil associado ao baixo peso no município de São Paulo, de 8,8, ainda pode ser considerado bastante inferior ao mesmo risco observado em Ribeirão Preto, que é de 17,4 . E, enquanto na comparação da mortalidade, os recém-nascidos de baixo peso do município de São Paulo chegam a ter uma ligeira menor probabilidade de óbito infantil $(305,5 \times 327,1)$, os recém-nascidos de peso superior a $3.000 \mathrm{~g}$ tèm no município de São Paulo quase duas vezes mais chance de morrer antes de completar o primeiro ano de vida $(34,4 \times 18,8)$.

Estas observações, além de situarem a aparente especificidade da relação peso ao nascer-óbito infantil no município, implicam a existência, no município de São Paulo, de fatores que, sem anular a decisiva influência biológica que o peso ao nascer exerce sobre a probabilidade de sobrevivência do recém-nascido, possam também estar contribuindo decisivamente, e independentemente do peso ao nascer, para a elerada mortalidade infantil do município.

Esta última possibilidade abre caminho para se encarar a segunda questão levantada por este estudo relativa ao real papel desempenhado pelo peso ao nascer na mortalidade infantil no município de São Paulo.

$\mathrm{O}$ ajuste da mortalidade do municipio de São Paulo à distribuição de peso ao nascer da Califórnia mostrou que 15 e $21 \%$. respectivamente, do excesso de mortalidade infantil e neonatal do município podem ser explicados apenas pelo relativo desfavorável desempento do peso ao nascer. Dada a universalidade biológica da influência do peso ao nascer sobre a probabilidade de óbito infantil, torna-se recomendável comparar a magnitude da participação do peso ao nascer na mortalidade de São Paulo com a observada $\mathrm{em}$ outras localidades.

Nos EUA, ajuste de mortalidade semelhante ao efetuado neste estudo, mostrou que, em 1960, 63\% do excesso de mortalidade infantil da população não branca americana, quase duas vezes superior a da população branca, poderia ser explicado apenas pelo pior desempenho do peso observado entre os não-brancos ${ }^{11}$. Com relação à mortalidade neonatal, praticamente $100 \%$ do excesso de mortalidade dos não brancos dever-se-ia exclusivamente ao seu pior peso ao nascer ${ }^{1 t}$. De mesma ordem de magnitude parece ser a participação do peso ao nascer na mortalidade infantil e neonatal das chamadas "áreas de pobreza" dos EUA ${ }^{13}$. Por outro lado, 85 a $90 \%$ do excesso de mortalidade neonatal dos EUA, frente a paises como a Suécia e a Holanda, poderiam ser atribuidos exclusivamente ao pior desempenho do peso ao nascer observado nos EUA ${ }^{12}$.

Em paises não desenvolvidos são escassos os estudos que procuram avaliar a influência do peso ao nascer sobre a mortalidade infantil, seja pela baixa confiabilidade dos registros vitais, seja pela inexistência de estudos sobre a distribuição do peso ao nascer na população. $\mathrm{Na}$ Guatemala, em Santa Maria Cauqué, Mata e col. ${ }^{6}$, através do estudo prospectivo de mais de $95 \%$ dos nascimentos ocorridos no período 1964-73, afirmam que os baixos valores alcançados pelo peso ao nascer nesta localidade explicariam em grande parte o seu excesso de mortalidade neonatal frente à população dos EUA. Peso a peso, referem esses autores, os recém-nascidos de Santa Maria Cauqué não teriam maior mortalidade neonatal do 
MONTEIRo, C.A. Estimativa dos coeficientes específicos de mortalidade infantil segundo peso ao nascer no municipio de São Paulo (Brasil). Rev. Saúde públ., S. Paulo, 15:603-10, 1981.

que os recém-nascidos de Baltimore nos EUA.

Vista comparativamente, portanto, a parti. cipação do peso ao nascer na mortalidade do municipio de São Paulo, ainda que ponderável, não parece ser tão importante quanto à registrada em outras localidades. Em trabalho anterior 10 chegou-se a conclusão semelhante, por meio do ajuste indireto da mortalidade infantil de 1976 à distribuição do peso ao nascer da população branca dos EUA. No trabalho presente, fica claro que esta menor importância decorre fundamentalmente de un coeficiente de mortalidade infantil particularmente elevado para quem nasce com mais de $3.000 \mathrm{~g}$ e de uma incidencia relativamente baixa de recém-nascidos de baixo peso, frente a que se esperaria tendo em conta a alta mortalidade.

O desdobramento natural do presente estudo implica a imediata revisāo das expectativas acerca dos efeitos da suplementação alimentar a gestantes no município de São Paulo ${ }^{17}$. Há suficientes elementos para se admitir como bastante improvável a repetição do sucesso obtido em aldeias da Guatemala onde, através de suplementação alimentar, reduziu-se à quase metade a mortalidade infantil 5. Há elementos, mesmo para não se aceitar no caso de São Paulo, a elevada prioridade que os autores da experiência guatemalteca pretendem para a suplementação de gestantes ${ }^{1}$.

Finalmente, é preciso nāo esquecer que no município de São Paulo, ao lado de relativas baixas incidências de baixo peso ao nascer, estão as mais altas incidências de óbitos por doença diarréica de que se tem noticia no continente ${ }^{15}$. Neste sentido, talvez mais útil do que voltar os olhos para algumas aldeias camponesas da Guatemala, fosse encarar a experiência nacional de países como Cubas e Costa Rica ${ }^{6}$, que, no prazo de poucos anos, reduziram de forma espetacular seus coeficientes de mortalidade infantil na vigência, o que é particularmente importante, de distribuição de peso ao nascer muito semelhante a do município de São Paulo.

MONTEIRO, C. A. [An estimate of the specific infant mortality rates by birth-weight in the city of S. Paulo (Brazil)]. Rer. Sacide públ., S. Paulo, 15:603-10, 1981.

ABSTRACT: By means of the birth-rate distribution found to exist during the Inter-American Investigation of Mortality in Childhood in $\mathbf{S}$. Paulo (1968-70) the infant death rate association with each birth-weight interval was estimated. The infant mortality rates were $305.5,50.2$ and 34.4 respectively for low birth-weight, deficient weight and weight above 3,000 grams. Compared with the rates found in California, USA, by the same Investigation, the infant mortality rates in $\mathbf{S}$. Paulo are higher, particularly for mortality associated with birth-weight of above 3,000 grams. The adjustment of infant mortality rates in $\mathrm{S}$. Paulo according to the birth-weight distribution found in California led to the conclusion that, at that reriod, only $15 \%$ of the excess of the infant mortality of $\mathrm{S}$. Paulo could be attributed exclusively to birth-weight. In the case of neonatal mortality, $21 \%$ of the mortality excess in $\mathrm{S}$. Paulo could be attributed to birth-weight.

CNITERMS: Infant mortality. Birth weight. S. Paulo, SP, Brazil. 
MONTEIRO, C.A. Estimativa dos coeficientes especificos de mortalidade infantil segundo peso ao nascer no municipio de São Paulo (Brasil). Rev. Saúde públ., S. Paulo, 15:603-10, 1981.

\section{REFERENCIAS RIBLIOGRÁFICAS}

1. DELGADO, H.; LECHTIG, A.; MARTORELL, R. \& KLEIN, R.E. Aplicación de técnicas simplificadas a programas de salud maternoinfantil: los aspectos nutricionales de alto riesgo. Bol. Ofic. sanit. panamer., 84:295-305, 1978.

2. LAURENTI. R. Alguns aspectos particulares referentes aos resultados da Investigação Interamericana de Mortalidade na Infância na área do projeto de São Pacio, Brasil. Bol. Ofic. sanit. panamer., 89 :1-14, 1975.

3. LAURENTI, R. Resultados e açōes apontadas pela Investigação Interamericana de Mortalidade na Infância no Brasil. Bol. Ofic. sanit. panamer., 82:344-60, 1977.

4. LECHTIG, A.; HABICHT, J.P.; DELGADO, H.: KLEIN, R.E.; YARBROUGH, C. \& MARTORELL, R. Effect of food supplementation during pregnancy on birth weight. Pediatrics, 56:508-20, 1975.

5. LECHTIG, A.; YARBROUGH, C.; DELGADO, H.; MARTORELL, R. \& KLEIN, R.E. Os efeitos de uma nutrição aperfeiçoada a partir da concepção da criança até os 3 anos de idade na mortalidade infantil. $J$. Pediat., 42:40-7, 1977.

6. MATA, L.; URRUTIA, J.J. \& MOHS, E. Implicaciones del bajo peso al nascer para la Salud Publica. Arch. latinoamer. Nutr., 27 (supl.) :198-222, 1977.

7. MCKOWN, T, \& GIBSON, J.R. Observations on all births $(23,790)$ in Birmingham, 1947. IV - "Premature birth". Brit. med. J., 3:513-7, 1981.

8. MINISTERIO DE LA SALUD PUBLICA DE CUBA. Informe Anual: 19\%8. Havana, 1979.

9. MONTEIRO, C.A. Contribuição para o estudo do significado da evolução do coeficiente de mortalidade infantil no município de São Paulo nas três últimas décadas (1950-1979). Rer. Saúde públ., S. Paulo, 16(1)1982, [no prelo]

10. MONTEIRO, C.A. Peso ao nascer e excesso de mortalidade infantil em São Paulo. Rev. Saúde públ., S. Paulo, 14:1-8, 1980.
11. MONTEIRO, C.A.; ISHII, M.; BENICIO, M.H. D'A \& REA, M.F. A distribuição uo peso ao nascer no município de São Paulo, Brasil. Rev. Saúde públ., $\mathbf{S}$. Paulo, 14:161-72, 1980.

12. NATIONAL CENTER FOR HEALTH STATISTICS. International comparisons of perinatal and infant mortality: the United States and six West European Countries. Vital Hlth Statist. Sér. $3(6)$ Mar. 1967.

13. NATIONAL CENTER FOR HEALTH STATISTICS. Selected rital and health statistics in poverty and nonpoverty areas of 19 large cities, United States, 1969-1971. Vital Hlth Statist. Ser. 21(26) Nov. 1975 .

14. NATIONAL CENTER FOR HEALTH STATISTICS, A study of infant mortality from linked records by birth weight, period of gestation and other variables: United States, 1960 liverbirth cohort. Vital Hlth Statist. 20 (12) May, 1972.

15. PUFFER, R.R, \& SERRANO, C.V. Caracteristicas de la mortalidad en la niñez. Washington, D.C., Organización Panamericana de la Salud, 1973. (OPAS Publ. Cient., 262.

16. SECRETARIA DE ESTADO DA SAÚdE. Instituto de Saúde. Prematuridade $e$ insuficiência ponderal do recém-nascido. aspectos médico-sanitarios. São Paulo, 1978. (Publ., 32. Série D, Divisão de Saúde Materna e da Criança, 13).

17. SUPLEMENTAÇÃO alimentar a gestantes para reduzir coeficiente de mortalidade infantil. Saúde, S. Paulo, 1(2): 1, Nov. 1976 .

18. SWAROOP, S. Introduction to health statistics. Edinburgh, E. \& S. Livingstone, 1960 .

19. TERUEL, J.R.; GOMES, U.A. \& NOGUEIRA, J.L. Investisación Interamericana de Mortalidad en la Niñez: peso al nascer en la región de Ribeirão Preto, São Paulo, Brasil. Bol. Ofic. sanit. panamer, $79 \cdot 139-45, \quad 1975$

Recebido para publicasão em 25/09/1981 Aprovado para publicação em 17/11/1981 